\title{
Viral Polypeptides and RNA of Defective Interfering Particles of IPN Virus Generated by Serial Undiluted Passaging in TO-2 Cells
}

\author{
Chu-Fang Lo*, Chung-Hsiung Wang**, Mau-Shain Lin** \\ and Guang-Hsiung Kou* \\ *Zoology Department, National Taiwan University, Taipei, Taiwan, Republic of China \\ **Biology Department, Fu-Jen University, Taipei, Taiwan, Republic of China
}

(Received January 11, 1991)

\begin{abstract}
HB-1 virus, a strain of infectious pancreatic necrosis virus (IPNV), was isolated from hard clam, Meretrix lusoria, with TO-2 cell line derived from tilapia ovary. The defective interfering (DI) particles of HB-1 virus could be generated by serial undiluted passaging. The polypeptides and RNA in virus particles of diluted and undiluted virus preparations were compared in order to investigate the nature of defective interfering particles involved in viral interference of HB-1 virus. Cesium chloride density gradient centrifugation was applied to purify virus particles of diluted and undiluted virus preparations. By comparing the polypeptides and RNA in virus particles of diluted and undiluted virus preparations, it was revealed that (I) the virus particles of undiluted virus preparation had smaller $\beta$ polypeptides than normal; (II) subgenomic RNA of large segment was found in virus particles of undiluted virus preparation. These two findings suggested that DI particles of HB-1 virus was a deletion mutant of standard virus.

This is the first report indicating the polypeptides and RNA possibly related to IPN virus DI particles.
\end{abstract}

Infectious pancreatic necrosis virus (IPNV) is an economically important pathogen of salmonid and nonsalmonid fishes (Dobos and Roberts, 1983). Several studies indicated that marine bivalves are also common hosts of IPNV (Dobos et al., 1979; Lo et al., 1988). Investigations conducted during the last two decades revealed that IPNV contains a double-stranded (ds) and segmented genome. By gel electrophoresis, the dsRNA genome could be separated into two segments. These two genome segments were present in equimolar amounts. The virusspecific polypeptides of IPNV fall into three size classes, large $(\alpha)$, medium $(\beta)$, and small $(\gamma)$. The minor polypeptide of large size class is encoded by the smaller RNA segment. The major polypeptides of medium and small size classes are encoded by the large RNA segment (Dobos and Roberts, 1983).

The interference phenomenon exhibited by IPNV has been described by several authors (Ahne, 1977; Hedrick and Fryer, 1982; Hedrick et al., 1978; Kennedy and MacDonald, 1982; Lo et al., 1990; MacDonald, 1978; MacDonald and Kennedy, 1979; MacDonald and Yamamoto, 1978; Malsberger and Cerini, 1963; Nicholson and Dunn, 1974). Most of the above studies indicated that defective interfering (DI) particles were involved in IPNV viral interference. In these studies, attempts were made to separate DI particles from the standard virus, but there were no available methods to achieve this purpose. Thus further analysis of biochemical properties of IPNV DI particles must await better purification methods. However, DI particles can be generated by serial undiluted passaging. Lo et al. (1990) observed the differences in the polypeptide composition of virions from serial diluted and undiluted prssaging of a strain of IPNV. The truncated $\beta$ polypeptides were regarded to be specific polypeptides produced by DI paritcles generated by serial undiluted passaging. The present studies showed that the comparison of the polypeptides and genomic 
RNA of virus particles produced by serial undiluted and diluted passaging of IPNV was a promissing method to investigate the nature of IPNV DI particles and also showed that the changes in proteins and RNA of virus particles due to serial undiluted passaging provided a possible method to elucidate the viral interference mechanism of IPNV mediated by DI particles.

\section{Materials and Methods}

\section{Cells and Viruses}

TO-2 cells, derived from tilapia ovary were propagated in Leibovitz's L-15 medium supplemented with either $2.5 \%$ fetal bovine serum for virus propagation or $5 \%$ for routine subculture of cells (Chen et al., 1983). The cells were incubated at $28^{\circ} \mathrm{C}$ for growth or at $20^{\circ} \mathrm{C}$ for virus propagation.

HB-1 clam virus, a strain of IPN virus (AB strain) isolated from the gills of Meretrix lusoria (Lo et al., 1988), was used in the present studies. Virus stock was prepared by five rounds of limiting dilution in TO-2 cells.

Serial undiluted passaging of $\mathrm{HB}-1$ virus was initiated by infecting TO- 2 cells $\left(1 \times 10^{7}\right.$ cells $)$ with $1 \mathrm{~m} l$ of undiluted virus stock. The passages were made at 7 day intervals. For the subsequent passages, $1 \mathrm{~m} l$ membrane $(0.22 \mu \mathrm{m})$ filtered culture fluid from previous passage was used as virus inoculum. For every passage, the infection was done by absorbing the virus inoculum to the cells for $1 \mathrm{~h}$ at $25^{\circ} \mathrm{C}$. The monolayer was washed three times to remove the unabsorbed virus, and then $5 \mathrm{ml}$ of fresh L-15 medium plus 2.5\% FBS was added per culture. Cultures were incubated at $20^{\circ} \mathrm{C}$ for 7 days. Serial diluted passaging of HB-1 virus was also done at the same time. Instead of undiluted virus, the virus inoculum for the absorption of the cells was at a dilution of $10^{-4}$.

Purification of Virus Particles Produced by Undiluted and Diluted Passaging with $\mathrm{CsCl}$ Gradient

Virus particles from passage 40 and 50 of undiluted or diluted $\left(10^{-4}\right)$ passaging were purified separately in $\mathrm{CsCl}$ gradient with a procedure described by Chang et al. (1978) with some modifications. Fractions were collected from the bottom for the analyses of viral polypeptide composition, and genomic RNA segments as described in the following.

\section{Infectivity and Interfering Ability of Purified Virus Particles Produced by Undiluted and Di- luted Passaging}

The infectivity was determined by TCID $_{50}$ assay. The protection was expressed as interference dose (ID). Briefly, $0.1 \mathrm{~m} l$ of appropriate dilution of virus samples of fractions collected from $\mathrm{CsCl}$ gradient at 10 -fold dilution was added onto TO- 2 cells in 96 well microplates following the same procedures done for $\mathrm{TCID}_{50}$ assay. Then, $0.1 \mathrm{ml}$ of virus prepared by five low-multiplicity rounds of amplification, was added to each well, which was supposed to produce lytic infection. The multiplicity of infection is $0.1 \mathrm{TCID}_{50} /$ cell. The interference dose is reciprocal of endpoint dilution of virus samples which protected $50 \%$ of cells from lytic infection. The buoyant density of each fraction was calculated from their refractive index.

Comparison of Polypeptides of Purified Virus Particles Produced by Serial Undiluted and Diluted Passaging

Polypeptides in each fraction collected from $\mathrm{CsCl}$ gradient were analyzed with $10 \%$ polyacrylamide gel electrophoresis and stained with silver stain.

Comparsion of RNA of Purified Virus Particles Produced by Serial Undiluted and Diluted Passaging

The fractions of passage 40 containing either mature or immature virus particles were pooled and pelleted for RNA analysis by $10 \%$ SDS polyacrylamide gel. The procedure was similar to that of Hedrick et al. (1985).

\section{Results}

Purification of Virus Particles Produced by Undiluted and Diluted Passaging

Cesium chloride $(\mathrm{CsCl})$ density gradient centrifugation was applied in the present studies to purify virus particles with different buoyant density. There were only two bands formed at the density of 1.30 and $1.34 \mathrm{~g} / \mathrm{ml}$, respectively 
in $\mathrm{CsCl}$ gradient for virus sample obtained from serial diluted passaging. For virus sample obtained from serial undiluted passaging, two major andfive minor bands were observed. The major bands banded also at density of 1.30 and 1.34 $\mathrm{g} / \mathrm{ml}$ in $\mathrm{CsCl}$ gradient. Three out of five minor bands were distributed between two major bands. Other two minor bands were located at near either upper part of the lighter major band or lower part of heavier major band. According to the thickness of bands, it was concluded that serial undiluted passaging favored the formation of virus particles banded at a density of $1.30 \mathrm{~g} / \mathrm{ml}$ in $\mathrm{CsCl}$ gradient.

Infectivity and Interfering Ability of Purified Virus Particles Produced by Serial Undiluted

\section{and Diluted Passaging}

Virus particles of passage 40 in $\mathrm{CsCl}$ gradient were collected from the bottom into fractions. The volume of each fraction was about $500 \mu l$. No attempt has been made in the present study to separate each band in $\mathrm{CsCl}$ gradient into different fractions. The fractions were assayed for infectivity and interfering ability as described in Materials and Methods section. Both virus samples obtained from multiple undiluted or diluted passage showed a peak of infectivity at fraction 7. Virus particles in these two fractions have a buoyant density of $1.33 \mathrm{~g} / \mathrm{ml}$ in $\mathrm{CsCl}$.

The virus titer of fraction 7 was $10^{10.2}$ $\mathrm{TCID}_{50 / 0.1 \mathrm{~m} l}$ for multiple diluted passage and $10^{9.8} \operatorname{TCID}_{50 / 0.1 \mathrm{~m} l}$ for multiple undiluted pas-

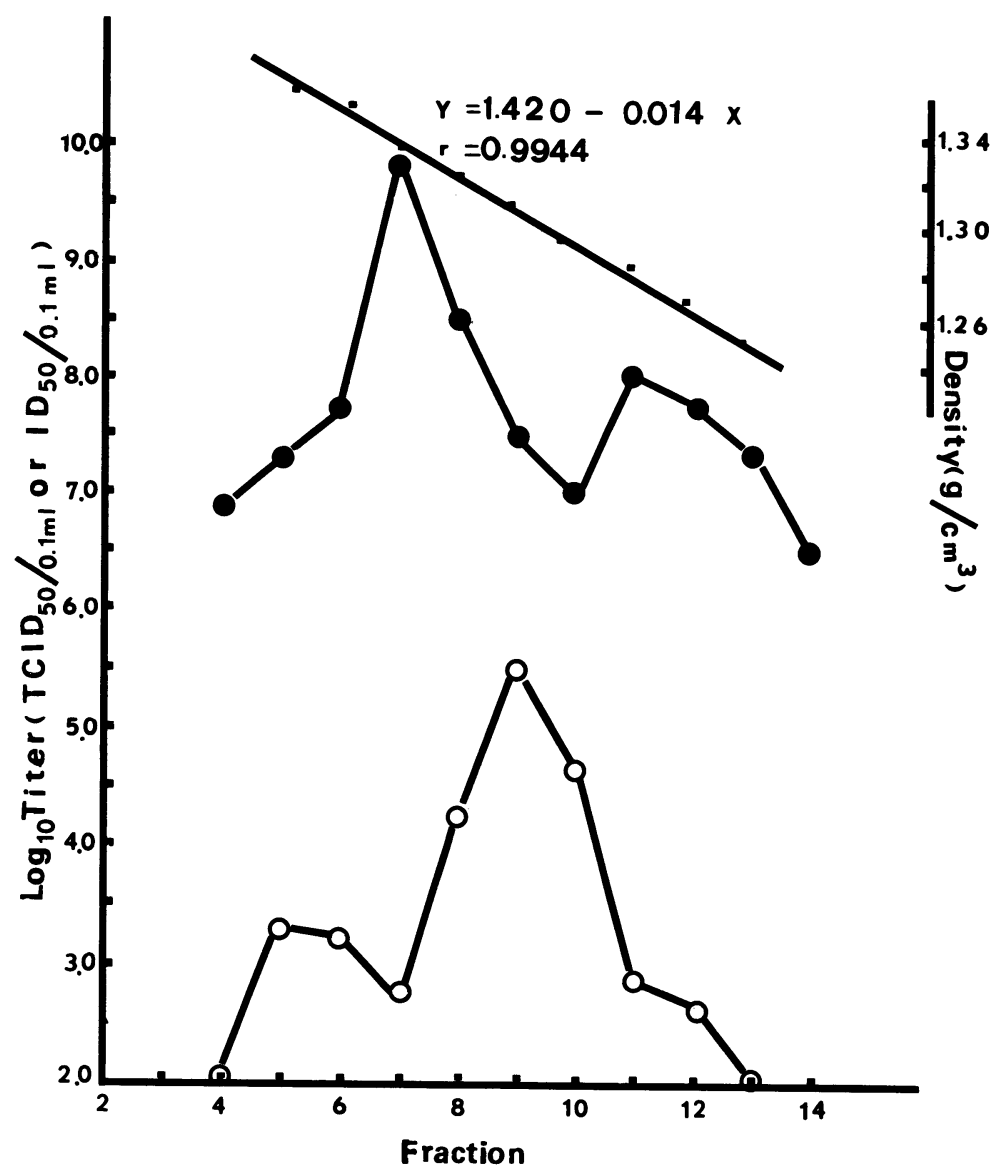

Fig. 1. Viral infectivity (๑) expressed as $\operatorname{TCID}_{50 / 0.1 \mathrm{~m} l}$ and viral interference $(\bigcirc)$ expressed as ID ${ }_{50 / 0.1 \mathrm{~m} l}$ in $\mathrm{CsCl}$ fractions of passage 50 undiluted viral preparation. 
sage.

All fractions of virus sample from multiple diluted passage did not show any viral interference, whereas the fraction 8 to fraction 10 of virus sample from multiple undiluted passage revealed a strong viral interference (Fig. 1). The interference titer ranged from $10^{4 \cdot 3}$ to $10^{5 \cdot 6}$ $\mathrm{ID}_{50 / 0.1 \mathrm{~m} l}$. The predominant peak of interference occurred at $1.30 \mathrm{~g} / \mathrm{ml}$ (fraction 9). Because the viral interference was observed only in fractions collected from virus sample prepared by multiple undiluted passage, we concluded that DI particles were generated due to multiple undiluted passage. The fact that the peak of infectivity and interference occurred at different fractions indicated that a physical difference between the DI particles and standard virus did exist.

\section{Polypeptides of Purified Virus Particles Produced} by Serial Undiluted and Diluted Passaging

The structural polypeptides of passage 50 virus particles were analyzed using polyacrylamide gel electrophoresis. The relative mobil- ities of the structural polypeptides for the virus particles of passage 50 in 5 fractions at densities of 1.30 to $1.34 \mathrm{~g} / \mathrm{ml}$ in $\mathrm{CsCl}$ gradient for undiluted and diluted virus preparations were shown in Figs. 2 and 3. Three size classes of viral polypeptides were detectable for virus particles from serial diluted or undiluted pasasging. In the $\alpha$ and $\gamma$ groups of viral polypeptides, no differences were observed in virus particles produced by serial undiluted and diluted passaging. Differences were observed only in the $\beta$ group (Figs. 3 and 4 ). Two polypeptides, VP 47 and VP 48 (molecular mass, 47,000 and 48,000 daltons (d)) were found in $\beta$ group of mature virions from serial undiluted passaging. VP 47 greatly exceeded in amount to VP 48. Both were smaller than VP $49(49,000 \mathrm{~d})$, the only one polypeptide found in the $\beta$ group of mature virions from serial diluted passaging (Figs. 3 and 4). The ratio of VP 47 to VP 48 increased as the number of passage increased (Fig. 3).

The polypeptide composition of immature virions which banded at light buoyant density,

A
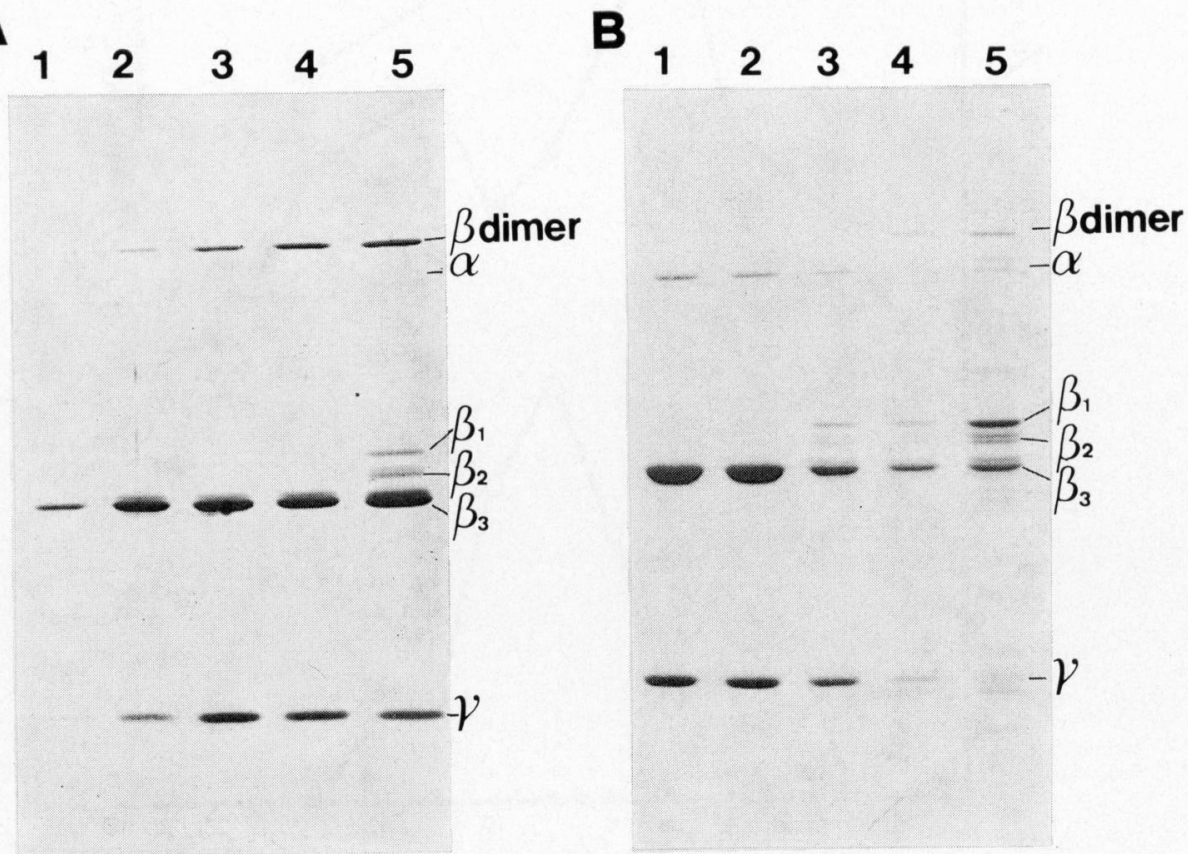

Fig. 2. Polypeptide profiles of passage 50 virus particles in 5 fractions at densities of 1.34 to $1.30 \mathrm{~g} / \mathrm{m} l$ (lane 1 to lane 5 ) in $\mathrm{CsCl}$ gradient. A, undiluted virus preparation: $\mathrm{B}$, diluted virus preparation. 


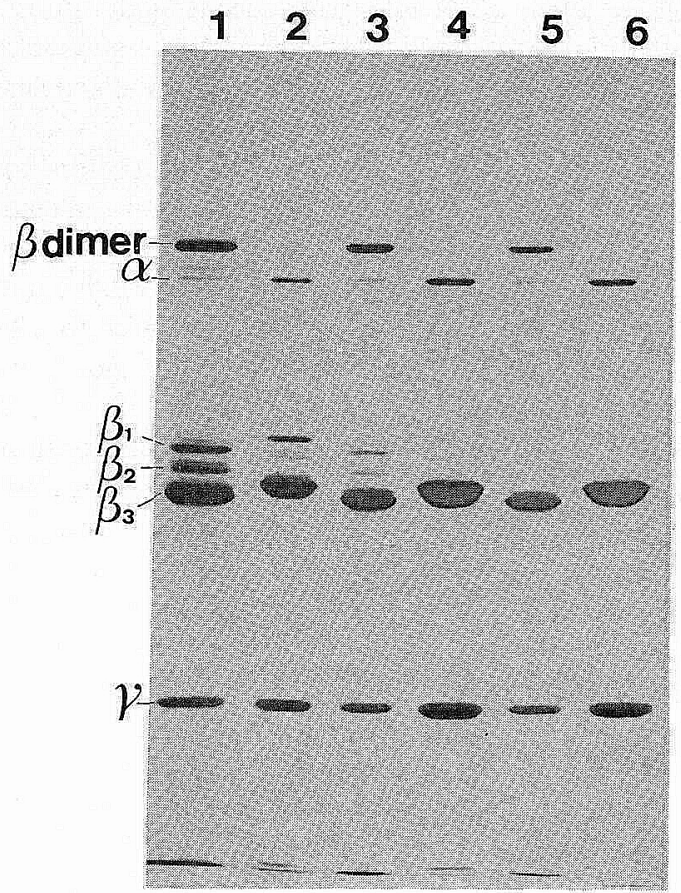

Fig. 3. Comparisons in polypeptide profiles of diluted and undiluted virus preparations of passage 50 in fractions at densities of 1.30 to $1.34 \mathrm{~g} / \mathrm{m} l$ (lane 1 to lane 6 ) in $\mathrm{CsCl}$ gradient. Lane 1, 3, 5: undiluted virus preparation; Lane 2, 4, 6: diluted virus preparation.

showed that differences in the primary gene product of $\beta$ group were also observed in samples of serial undiluted and diluted passaging (Figs. 3 and 4). For primary gene product, VP $58(58,000$ d.) was found in virus samples from serial diluted passaging while VP 57 and VP $56(57,000$ d. and 56,000 d.) were found in virus samples from serial undiluted passagig. Furthermore, the dimer of $\beta$ polypeptide appeared in relatively large amount in immature and mature virus particles of undiluted virus preparation (Fig. 3). In contrast, the $\beta$ dimer was never found in mature virions of diluted virus preparation. All the present results confirmed that in $\beta$ group, the virus particles of serial undiluted passaging had smaller viral polypeptides than those of serial diluted passaging.

\section{$R N A$ in Purified Virus Particles Produced by}

A
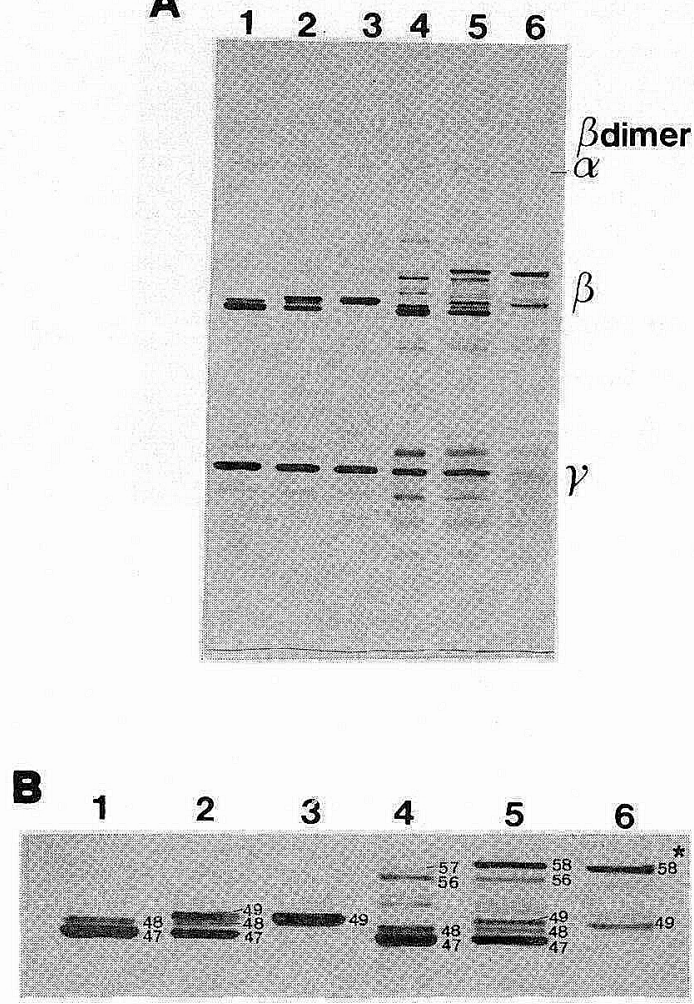

Fig. 4. Polypeptide profiles of purified passage 40 virus particles of undiluted and diluted virus preparations. $\mathrm{A}, \alpha, \beta$ and $\gamma$ groups of viral polypeptides; $B$, high magnification of $\beta$ group of viral polypeptides; lane 1 , virus particles of undiluted virus preparation, banded at $1.34 \mathrm{~g} / \mathrm{m} l$ in $\mathrm{CsCl}$; lane 2 , mixed virus particles of diluted and undiluted virus preparations, banded at $1.34 \mathrm{~g} / \mathrm{ml}$ in CsCl; lane 3, virus particles of diluted virus preparation, banded at $1.34 \mathrm{~g} / \mathrm{ml}$ in $\mathrm{CsCl}$; lane 4 , virus particles of undiluted virus preparation, banded at $1.30 \mathrm{~g} / \mathrm{ml}$ in $\mathrm{CsCl}$; lane 5 , mixed virus particles of diluted and undiluted virus preparations, banded at $1.30 \mathrm{~g} / \mathrm{m} l$ in $\mathrm{CsCl}$; lane 6 , virus particles of diluted virus preparation, banded at $1.30 \mathrm{~g} / \mathrm{ml}$ in $\mathrm{CsCl}$

* The molecular mass of the polypeptides is indicated in kilodaltons.

\section{Serial Undiluted and Diluted Passaging}

Virus particles in passage 40 of serial undiluted and diluted passaging were purified and subjected to $\mathrm{CsCl}$ gradient centrifugation. The 


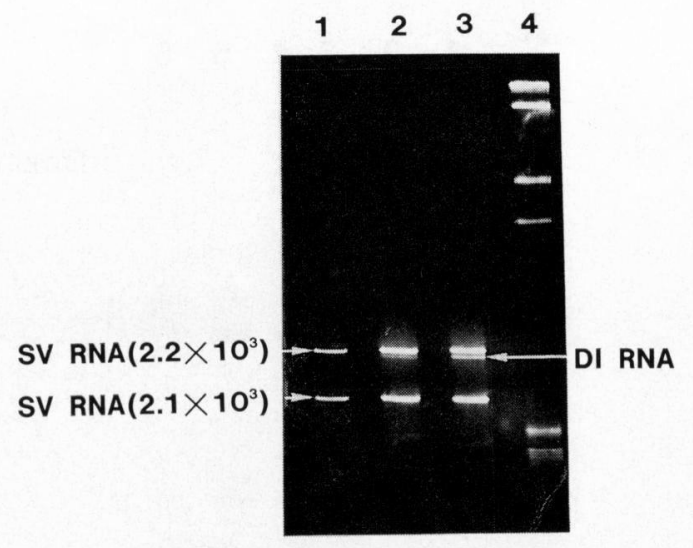

Fig. 5. Comparisons of RNA extracted from passage 40 virus particles of diluted and undiluted virus preparations. Lane 1, diluted virus preparation; lane 2, mixture of diluted and undiluted vilus preparations; lane 3 , undiluted virus preparation; lane $4, \lambda$ phage DNA Hindi III fragments.

* The size of RNA is indicated in kilodaltons.

SV: standard virus; DI: defective interfering particle.

fractions at densities from $1.30 \mathrm{~g} / \mathrm{m} l$ to $1.34 \mathrm{~g}$ / $\mathrm{m} l$ in $\mathrm{CsCl}$ gradient were pooled for RNA analysis (Fig. 5). Two standard genomic RNA segments $\left(2.2 \times 10^{6}\right.$ and $2.1 \times 10^{6} \mathrm{~d}$. $)$ were present in both virus preparations while subgenomic RNA of the large segment was observed only in virus preparation of serial undiluted passaging. The subgenomic RNA was observed to be in relatively smaller concentration when compared to normal genomic RNA segments. Approximately, 37 nucleotides of large RNA segment were deleted for subgenomic RNA. No subgenomic RNA of small RNA segment was detectable in these virus preparations. The data indicated that DI particles involved in viral interference of HB-1 virus was a deletion mutant of the standard virus.

\section{Discussion}

DI particles of $\mathrm{HB}-1$ virus was considered to be a major controlling element of virus infectivity. HB-1 virus DI particles seemed to be constantly generated at low levels by infectious virus and only amplify to interfering levels when virus was passaged at high multiplicity of infection. The abundance of standard virus favored the formations of DI particles (Lo et al., 1990).

It is surprising to find that in addition to VP 47, VP 57 and VP 48 were present instead of VP 58 and VP 49 in virions from serial undiluted passaging. The absence of VP 58 and VP 49 may be due, as commented here, to the incomplete glycosylation of normal $\beta$ polypeptides in response to the presence of VP 47. There was no data to show the relationship between the VP 47 and viral interference of HB-1 virus, but the present studies strongly suggested that VP 47 appeared only in virus preparation of serial undiluted passaging, which favored the generation of DI particles. Thus it is reasonable to suggest that DI particles generated by serial undiluted passaging were responsible to the presence of VP 47.

The present studies also indicated that DI particles of HB-1 virus contained subgenomic RNA of large genomic RNA segment due to an unknown mechanism. It is likely that the deletions of DI RNA occurred in a part of the genome essential for infectivity, which resulted in the decrease of yields in infectious virus particles. Due to the consistency of the presence of DI RNA and VP 47 in virus samples from different sets of serial undiluted passaging, it is suggested here that DI RNA was formed by deleting some nucleotide sequences of standard virus (SV) RNA at specific regions. In the other word, the deletions did not seem to occur randomly. From the size of DI polypeptides and DI RNA, it was estimated that approximately 12 amino acids of SV $\beta$ polypeptides were truncated. In virus samples from serial undiluted passaging, the ratio of VP 47 to VP 48 was not consistent with the ratio of DI RNA to SV RNA (Figs. 4 and 5). The high ratio of VP 47 to VP 48, as well as the low ratio of DI RNA to SV RNA implied that (i) DI RNA produced its gene product at high speed, (ii) the production of VP 48 or VP 49 by SV RNA was inhibited by DI RNA or DI RNA gene product, (iii) the interference seemed to occur not at replication but at transcription or translation level, and (iv) the reduction of infectious virus particles was because of the loss of normal 
capsid protein, which is important for infectivity, rather than the preferential replication of DI particles. However, further studies would be required to elucidate the mechanism in the viral interference of $\mathrm{HB}-1$ virus.

\section{Acknowledgement}

This research was supported by National Science council (NSC 80-0418-B002-10), ROC.

\section{References}

Ahne, W. (1977): Persistent infection in CHSE-214 cells with IPN virus isolated from pike (Esox lucius). Bull. Off. Int. Epizoot., 87, 415-417.

Chang, N. R., D. MacDonald, and T. Yamamoto (1978): Purification of infectious pancreatic necrosis (IPN) virus and comparison of polypeptide composition of different isolates. Can. J. Microbiol., 24, 19-27.

Chen, S.-N., S.-C. Chi, Y. Ueno, and G.-H. Kou (1983): A cell line derived from Tilapia ovary. Fish Pathol., 18, 13-18.

Dobos, P. and T. E. Roberts (1983): The molecular biology of infectious pancreatic necrosis virus: a review. can. J. Microbiol., 29, 377-384.

Dobos, P., B. J. Hill, and R. Hallett (1979): Biophysical and biochemical characterization of five animal viruses with bisegmented double-stranded RNA genomes. J. Virol., 32 (20), 593-605.

Hedrick, R. P. and J. L. Fryer (1982): Persistent infections of salmonid cell lines with infectious pancreatic necrosis virus (IPNV): a model for the carrier state in trout. Fish Pathol., 16, 163-172.

Hedrick, R. P., W. D. Eaton, J. L. Fryer, Y.-C. Hah, J.-W. Park, and S.-W. Hong (1985): Biochemical and serological properties of birnaviruses isolated from fish in Korea. Fish. Pathol., 20, 463-468.

Hedrick, R. P., J. C. Leong, and J. L. Fryer (1978): Persistent infections in salmonid fish cells with infectious pancreatic necrosis virus (IPNV). $J$. Fish Dis., 1, 297-301.

Kennedy, J. C. and R. D. MacDonald (1982): Persistent infection with infectious pancreatic necrosis virus mediated by defective-interfering (DI) virus particles in a cell line showing strong interference but little DI replication. J. Gen. Virol., 58, 361-371.

Lo, C.-F., Y.-W. Hong, S.-Y. Huang, and C.-H. Wang (1988): The characteristics of the virus isolated from the gill of clam, Meretrix lusoria. Fish Pathol., 23, 147-196.

Lo, C.-F., M.-S. Lin, S.-M. Liu, C.-H. Wang, and G.-H. Kou (1990): Viral interference in TO-2 cells infected with IPN virus isolated from clam, Meretrix lusoria. Fish Pathol., 25, 133-140.

MacDonald, R.D. (1978): Ringed plaque formation in infectious pancreatic necrosis correlates with defective interfering particle producction. J. Gen. Virol., 41, 523-628.

MacDonald, R. D. and J. C. Kennedy (1979): Infectious pancreatic necrosis virus persistently infects chinook salmon embryo cells independent of interferon, Virology, 95, 260-264.

MacDonald, R. D. and T. Yamamoto (1978): Quantitative analysis of defective interfering particles in infectious pancreatic necrosis virus preparation. Arch. Virol., 57, 77-89.

Malsberger, R. G. and C. P. Cerini (1963): Characteristics of infectious pancreatic necrosis virus. J. Bacteriol., 86, 1283-1287.

Nicholson, B. L. and J. Dunn (1974): Homologous viral interference in trout and Atlantic salmon cell cultures infected with infectious pancreatic necrosis virus. J. Virol., 14, 180-182. 